\title{
INTRAMESOSIGMOID HERNIA PRESENTING AS INTESTINAL OBSTRUCTION
}

\author{
Nadeem Ahmad ${ }^{1}$, Nameer Faiz², Rinku Kumari ${ }^{3}$, Krishna Gopal ${ }^{4}$
}

${ }^{1}$ Senior Resident, Department of General Surgery, Indira Gandhi Institute of Medical Sciences, Patna, India. ${ }^{2}$ Senior Resident, Department of General Surgery, Indira Gandhi Institute of Medical Sciences, Patna, India.

${ }^{3}$ Senior Resident, Department of General Surgery, Indira Gandhi Institute of Medical Sciences, Patna, India.

${ }^{4}$ Associate Professor, Department of General Surgery, Indira Gandhi Institute of Medical Sciences, Patna, India.

HOW TO CITE THIS ARTICLE: Ahmad N, Faiz N, Kumari R, et al. Intramesosigmoid hernia presenting as intestinal obstruction. J. Evolution Med. Dent. Sci. 2017;6(47):3673-3675, DOI: 10.14260/Jemds/2017/793

\section{PRESENTATION OF THE CASE}

A 55-year-old male was admitted in the surgical emergency department with a 2-day history of central abdominal pain, abdominal distension and non-passage of flatus and stool with single episode of vomiting. Patient had no previous surgical history. Patient had stable vitals and general condition. Abdominal examination revealed mildly distended abdomen mainly around umbilicus, but otherwise soft with slight tenderness. Bowel sounds were sluggish. No inguinal hernias were present. Erect abdominal x-ray revealed 4 - 5 air-fluid levels without much bowel dilatation (Fig. 1).

Conservative management was attempted for 24 hours with NG tube and intravenous fluid resuscitation, but the patient's condition remained unchanged. Exploratory laparotomy was performed and intraoperative findings showed dilated proximal small bowel loops. The bowel loops were traced and transition zone of dilated and collapsed bowel was found. Segment of small bowel 1 feet proximal to ileocaecal junction was found herniating through a defect in the left surface of sigmoid mesocolon (Fig. 2). Herniated bowel was pulled out gently after carefully dissecting and dilating the margins of hernia defect. The incarcerated part of the bowel was $8 \mathrm{~cm}$ in length with developing stricture due to pressure from hernia defect margins (Fig. 3). Bowel was viable and showed much improvement in colour and peristalsis after hot fomentation, so no bowel resection was required. An oval hernia defect was present only on the left leaf of sigmoid mesocolon around $2.5 \mathrm{~cm}$ in size (Fig. 4), which was repaired with absorbable sutures.

Patient improved symptomatically and was discharged. Patient was followed up for 1 year and had no complaints or complications.

\section{DIFFERENTIAL DIAGNOSIS}

- Bands and adhesions.

- Intussusception.

- Volvulus.

\section{CLINICAL DIAGNOSIS}

Intestinal obstruction due to internal hernia.

Financial or Other, Competing Interest: None.

Submission 08-05-2017, Peer Review 02-06-2017,

Acceptance 07-06-2017, Published 12-06-2017.

Corresponding Author:

Dr. Nadeem Ahmad,

Flat-312, Tara Towers Apartment,

P. O. Shastri Nagar,

Patna-800023,

Bihar, India.

E-mail: ahmad.drnadeem@gmail.com

DOI: $10.14260 /$ jemds $/ 2017 / 793$

\section{PATHOLOGICAL DISCUSSION}

The most common cause of intestinal obstruction is postoperative adhesions. Internal hernia is the protrusion of intra-abdominal viscera through peritoneal and mesenteric orifices or defects within the peritoneal cavity. Internal hernia is a rare cause of Intestinal Obstruction accounting for $5.8 \%$ of the cases and paraduodenal hernia representing $50 \%$ of all the cases.[1],[2]

Sigmoid mesocolic hernia is a rare type of internal hernia with intramesosigmoid hernia being the rarest.[3],[4],[5]

Surgical approach through either exploratory laparotomy or laparoscopic approach is the standard of treatment. We present a case of Intramesosigmoid hernia causing small bowel obstruction, which was managed by surgical laparotomy.

Intestinal obstruction due to internal hernia is rare (5.8\%) and even rarer is the internal hernia involving the sigmoid mesocolon accounting for only $5 \%$ of the internal hernia cases.[6]

Benson et al[7] classified sigmoid hernia into three types(Fig. 5)

1. Intersigmoid Hernia: Herniation into an intersigmoid fossa, situated at the attachment of the lateral aspect of the sigmoid mesocolon.

2. Transmesosigmoid Hernia: Incarceration of intestinal loops through an isolated, oval defect in the sigmoid mesocolon.

3. Intramesosigmoid Hernia: A congenital, oval defect unrelated to the intersigmoid fossa is present in the lateral peritoneal surface of the mesocolon and herniation occurs. A normal fusion fascia is present and the right leaf is intact in this setting.

In our case, it was intramesosigmoid hernia (Type 3) according to the Benson et al classification. Benson et al was the first to describe a patient with intramesosigmoid hernia in 1963.[7] An intramesosigmoid hernia is predominantly seen in males with a peak incidence in $6^{\text {th }}$ decade of life.[7,8-12]

Although, pre-operative diagnosis is difficult, but CT scan of abdomen can suggest diagnosis with findings like a cluster of dilated fluid filled small bowel loops entrapped in the left posterior and lateral aspect of sigmoid colon with mesenteric vessel engorgement and dilated proximal bowel loops. ${ }^{[13]}$

\section{DISCUSSION OF MANAGEMENT}

Although, small bowel obstruction due to mesocolic internal hernia is rare, but should be suspected when the patient has no previous surgical history, tuberculosis or external hernia. Internal hernia is an uncommon cause of small bowel obstruction. Internal hernia is difficult to diagnose clinically. 
Pre-operative diagnosis is uncommon, but CT may suggest the diagnosis. It is interesting to know that congenital internal hernia is more common in paediatric age group. ${ }^{[14]}$ Our case was a 55-year-old male, so high index of suspicion and early surgical exploration is recommended in such cases of diagnostic dilemma to reduce the morbidity and mortality.

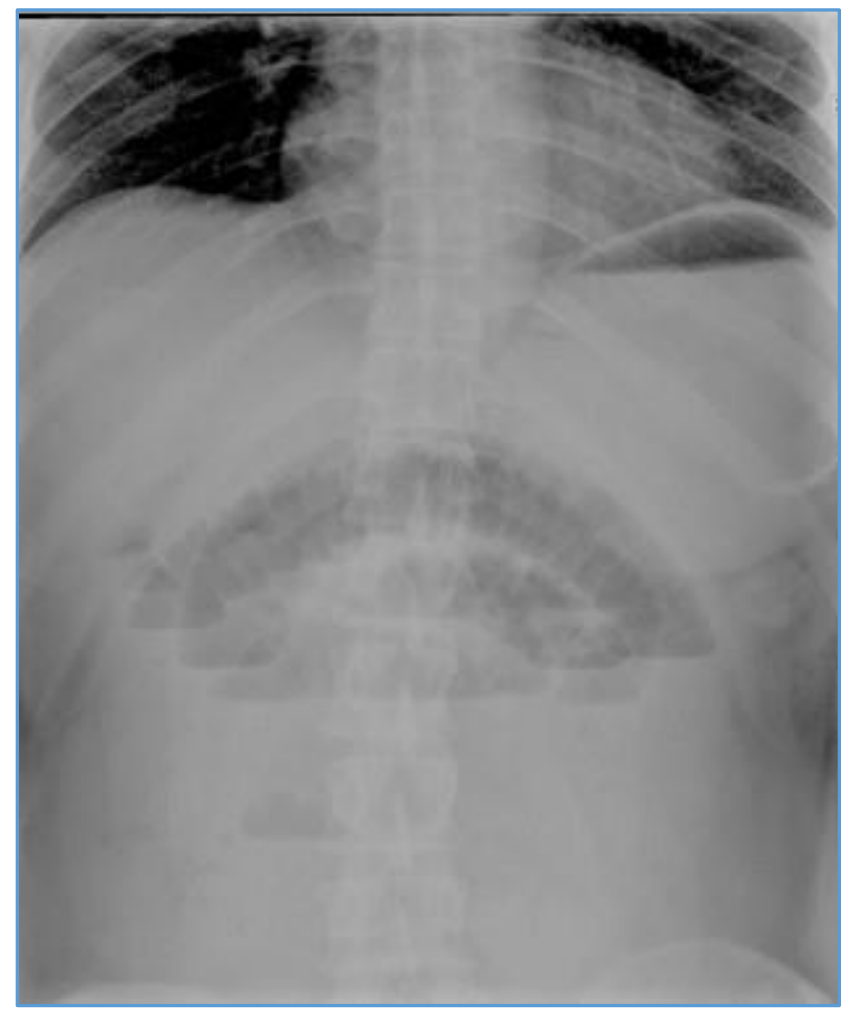

Figure 1. X-Ray Abdomen Erect showing Multiple Air-Fluid Levels and Dilated Small Bowel Loops

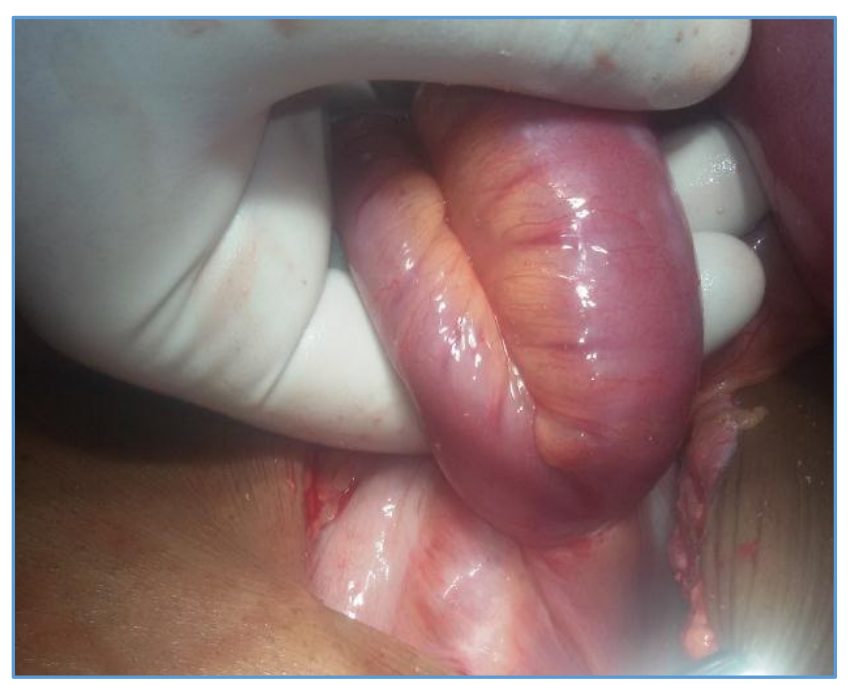

Figure 2. Small Bowel Loop Herniating through the Mesocolic Defect

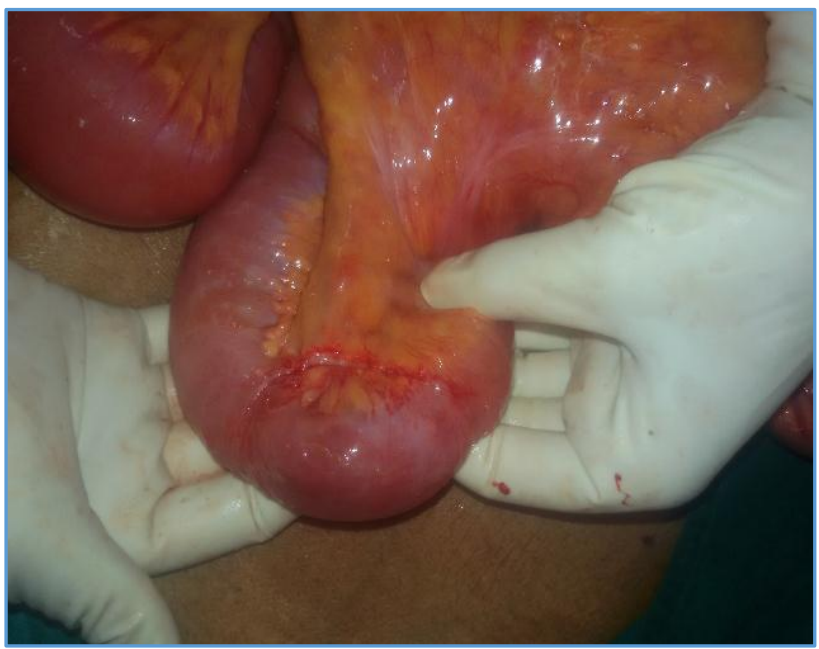

Figure 3. Incarcerated Bowel Loop Segment showing Stricture due to Pressure from Hernial Defect Margin

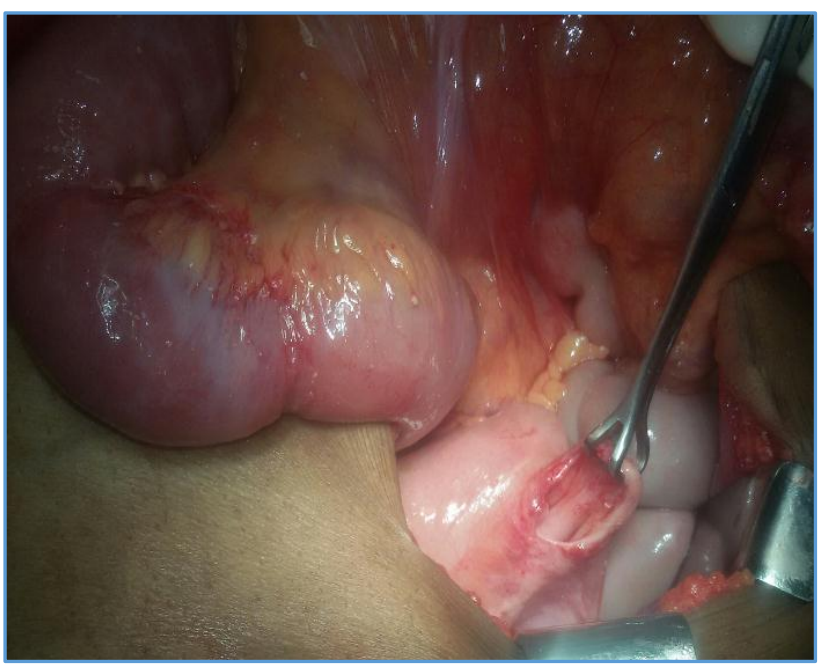

Figure 4. $2.5 \mathrm{~cm}$ Oval Hernial Defect on Left Leaf of Sigmoid Mesocolon

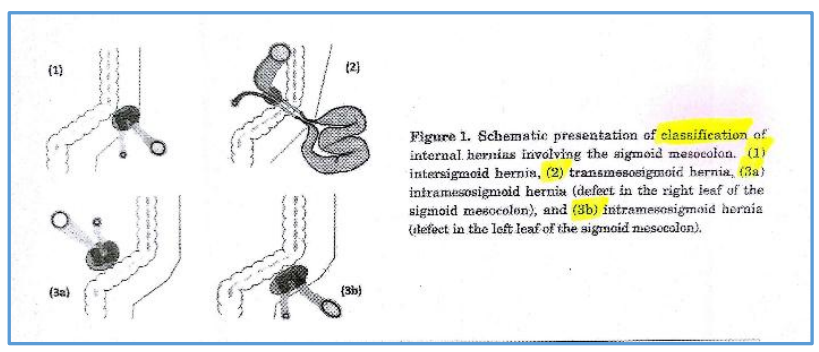

Figure 5. Benson et al Classification of Sigmoid Hernia

\section{FINAL DIAGNOSIS}

Intramesosigmoid hernia.

\section{REFERENCES}

[1] Zissin R, Hertz M, Gayer G, et al. Congenital internal hernia as a cause of small bowel obstruction: CT findings in 11 adult patients. $\mathrm{Br} \mathrm{J}$ Radiol 2005;78(933):796-802.

[2] Moran JM, Salas J, Sanjuan S, et al. Paramesocolic hernias: consequences of delayed diagnosis. Report of three new cases. J Pediatr Surg 2004;39(1):112-6. 
[3] Mieren VDG, de Gheldere C, Vanclooster P. Transmesosigmoid hernia: report of a case and review of the literature. Acta Chir Belg 2005;105(6):653-5.

[4] Jimmy J, Wani SV, Shetty VV, et al. Laparoscopic management of small bowel obstruction caused by a sigmoid mesocolic hernia. J Minim Access Surg 2011;7(4):236-8.

[5] Puri V, Bertellotti RP, Garg N, et al. Intramesosigmoid hernia: a rare type of congenital internal hernia. Hernia 2007;11(5):463-5.

[6] Martin LC, Merkle EM, Thompson WM. Review of internal hernias: radiographic and clinical findings. AJR Am J Roentgenol 2006;186(3):703-17.

[7] Benson JR, Killen DA. Internal hernias involving the sigmoid mesocolon. Ann Surg 1964;159(3):382-4.

[8] Kan H, Suzuki H, Takasaki H, et al. A case of an intramesosigmoid hernia. J Nippon Med Sch 2009;76(1):13-8.

[9] Yokota T, Yamada Y, Murakami Y, et al. Emergency abdominal surgery for sigmoid mesocolic hernia. Am J Emerg Med 2002;20(2):137-8.
[10] Taras A, Martinez R. Image of the month-quiz case. Intramesosigmoid hernia. Arch Surg 2009;144(12):1187-8.

[11] Murata Y, Miyata K, Yuasa N, et al. Case of intramesosigmoid hernia, nihon rinsho geka gakkai zasshi. Journal of Japan Surgical Association 2009;70(3):886-90.

[12] Isobe $H$, Takiguchi $M$, Hayashi $K$, et al. a case of intramesosigmoid hernia treated with laparoscopy, nihon rinsho geka gakkai zasshi. Journal of Japan Surgical Association 2007;68(2):447-51.

[13] $\mathrm{Yu} \mathrm{CY}, \mathrm{Lin} \mathrm{CC}, \mathrm{Yu}$ JC, et al. Strangulated Transmesosigmoid hernia: CT diagnosis. Abdom Imaging 2004;29(2):158-60.

[14] Janin Y, Stone AM, Wise L. Mesenteric hernia. Surg Gynecol Obstet 1980;150(5):747-54. 